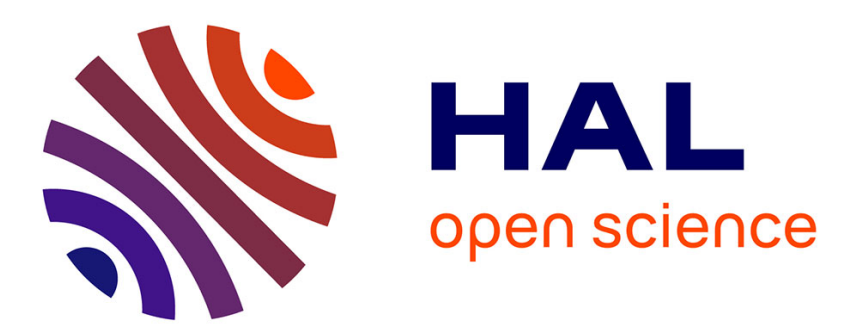

\title{
A Learning Approach for Robust Carrier Recovery in Heavily Noisy Visible Light Communication Antonio Costanzo, Valeria Loscrì
}

\section{To cite this version:}

Antonio Costanzo, Valeria Loscrì. A Learning Approach for Robust Carrier Recovery in Heavily Noisy Visible Light Communication. WCNC 2019 - IEEE Wireless Communications and Networking Conference, Apr 2019, Marrakesh, Morocco. hal-02022625

\section{HAL Id: hal-02022625 https://hal.science/hal-02022625}

Submitted on 18 Feb 2019

HAL is a multi-disciplinary open access archive for the deposit and dissemination of scientific research documents, whether they are published or not. The documents may come from teaching and research institutions in France or abroad, or from public or private research centers.
L'archive ouverte pluridisciplinaire HAL, est destinée au dépôt et à la diffusion de documents scientifiques de niveau recherche, publiés ou non, émanant des établissements d'enseignement et de recherche français ou étrangers, des laboratoires publics ou privés. 


\section{A Learning Approach for Robust Carrier Recovery in Heavily Noisy Visible Light Communication}

\author{
$1^{\text {st }}$ Antonio Costanzo \\ Inria Lille - Nord Europe \\ antonio.costanzo@inria.fr
}

\author{
$2^{\text {nd }}$ Valeria Loscri \\ Inria Lille - Nord Europe \\ valeria.loscri@inria.fr
}

\begin{abstract}
Visible Light Communication (VLC) exploits optical frequencies, diffused by usual LED lamps, for adding data communication features to illuminating systems. This paradigm has attracted a growing interest in both scientific and industrial community in the latter decade. Nevertheless, classical wireless communication mechanisms for physical and Medium Access Control (MAC) layers are hardly available for VLC, due to the massive external interference caused by sunlight. A correct signal carrier recover in high noise conditions represent a significant challenge. In this work, it is shown that the synchronization frame length affects the performance of the system in terms of Bit Error Ratio (BER). Since different external conditions require different minimum preamble lengths, we considered an Artificial Intelligence (AI) approach, based on multi-arm bandit formulation, for obtaining a low impact in both BER and goodput of the communication. A low-cost hardware VLC system, implementing a learning algorithm on a Frequency Shift Keying Modulation (FSK), has been designed and tested in different environmental conditions. Experimental results show that a proper choice of preamble length overcomes, in terms of BER and goodput, the classical approach based on fixed preambles.
\end{abstract}

Index Terms-Visible Light Communication, Carrier Recovery, Thompson Sampling, Multi-arm Bandit, Artificial Intelligence

\section{INTRODUCTION}

VLC presents several advantages compared to the RF wireless communication. First of all, visible light can be simultaneously exploited for illumination and communication and could be used in all the places where RF solutions are forbidden such hospitals, aircraft, etc. Several potential applications are envisaged for VLC such as vehicle to vehicle communication [1], underwater communication [2], robots in hospitals, efficient indoor geo-localization [3], etc. However, physical and MAC protocols, well assessed in RF contexts, are not suitable in the VLC domain. Indeed, specific features involved in the VLC paradigm, such as the fast changing external interference, need to be considered, in order to make the communication system robust and resilient. With this perspective, carrier recovery in a basic VLC system has been investigated for the first time in [4]. By analyzing the preamble length in a frame sent by the transmitter, the impact of the preamble length in terms of Bit Error Ratio (BER) and throughput has been evaluated. Results of this analysis were surprising, since different synchronization frame sizes impact in terms of carrier recovery precision and throughput. In this work, preamble choice is formulated as a multi-arm bandit problem and the most suitable preamble size changes according to external interference conditions. In order to validate the performance of the system, we implemented a low cost prototype and the algorithm has been implemented at the receiver side. An accurate analysis of the impact of the preamble length in different real conditions, and the implementation of the preamble variation, using as a multi-arm bandit problem with a Thompson sampling approach, represent the main contributions of this work. Indeed, evaluation performances show the effectiveness of our approach. The rest of the paper is organized as follow. Section II describes the system and the prototype. In section III, the analysis of the impact of synchronization frame length by motivating the design and implementation of an AI based approach. Section IV details the proposed algorithm and in Section V, experimental results are shown in order to validate the proposed approach. Finally, future research directions are outlined in Section VI.

\section{System Description}

In recent years, several VLC prototypes and different modulation techniques have been proposed [5], [6] . In order to reduce hardware costs, an interesting approach is the software defined approach, widely used in other contexts [7], [8]. Since most of signal operations are performed via software (modulation, demodulation, filtering, etc.), an extremely low cost hardware architecture has been employed and a high degree of flexibility has been achieved. The proposed system is represented by a couple of identical VLC communication devices, each one composed of the hardware equipment listed below.

- Transmitting front-end is made up of an array of 6 low power warm white leds (MCEEZW-A1, $1 \mathrm{~W} / \mathrm{m}^{2}$ ), a simple driving circuit and an Arduino Uno board. A $12 \mathrm{~V}$ power supply and a photo-diode dedicated to the reception of feedback control messages is also mounted on the same box.

- Receiving front-end is composed by a low cost photodiode (Centronic OSD15-5T), a trans-impedance amplifier and an Arduino Uno board. Since the photodiode is used in photovoltaic mode, no additional power supplies are needed.

- A personal computer is equipped by the commercial software LabView (National Instruments) for data elaboration. 
A picture, showing two VLC prototypes during experimental tests, is provided in Fig. 1.

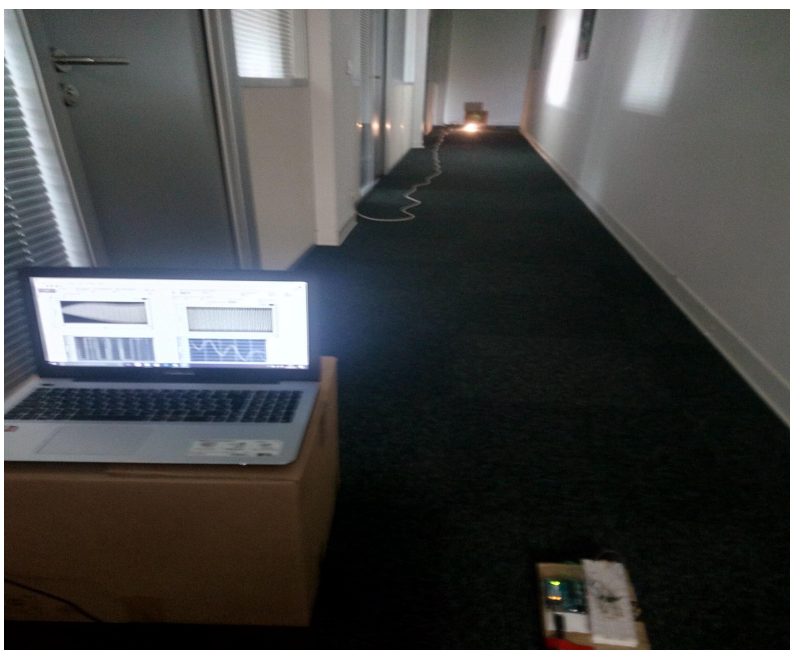

Fig. 1. Proposed VLC devices during measurements

Main operations, performed in the transmitting and receiving stages, are resumed in Fig. 2. A variable length preamble,
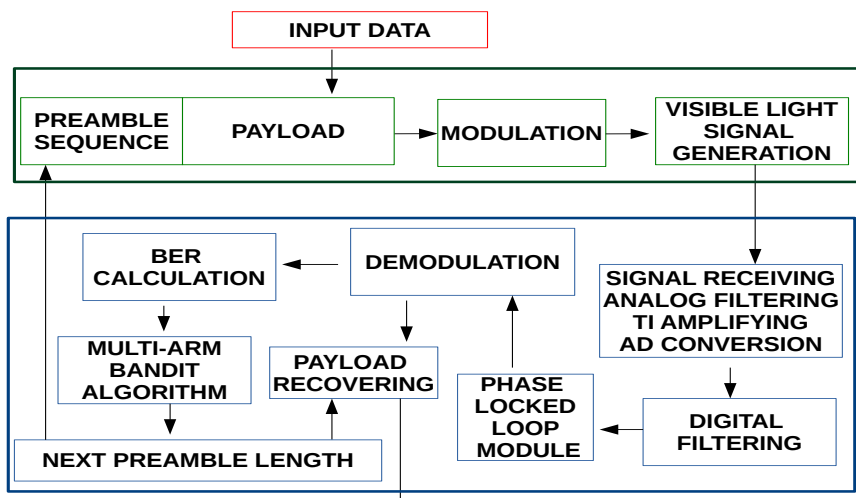

OUTPUT DATA

Fig. 2. Main operations performed by the proposed VLC system

corresponding to an alternate sequence of high and low levels, is appended to the payload and modulated using a Binary Frequency Shift Keying (BFSK) modulation, using ON-OFF voltage levels for controlling LED blinking [10], [11]. Once the signal has been received, a trans-impedance amplifying is performed in order to convert the current signal provided by the photodiode into a voltage signal, available to the other operations. A digital filtering block, composed of a sequence of adaptive filters, has been implemented. Once the signal is received and filtered, a central carrier detection is used to evaluate the preamble sequence and a phase locked loop is so performed in order to achieve a correct demodulation. At receiving stage, a real time BER analysis is performed and given in input to a multi-arm bandit based algorithm, which provides the length of the synchronization frame that will be used in the next transmission. Some preliminary experimental tests, showing how synchronization preamble length impacts on the Bit Error Ratio (BER) and throughput have been performed and shown in section III.

\section{Motivations}

\section{A. Issues}

Optical noise sources in real environments significantly affect performances of Visible Light communication schemes. Even if this aspect represents one of the main factors delaying an extensive diffusion of Visible Light Communication technology, relatively few authors in current literature have focused on the implementation of adaptive algorithms taking into account real time evolution of environmental conditions [9]. In order to correctly recover frequency, phase, and symbol timing of the carrier, a context aware system is developed in this work. It dynamically establishes the minimum synchronization frame size for an affordable carrier recovery regardless of the modulation scheme employed. Even if a frequency shift keying modulation scheme has been used in order to easily verify the correctness of the proposed algorithm, our proposed Thompson sampling algorithm may be employed, without loss of generality, with OOK, PSK,PPM and OFDM. In order to avoid carrier detection failures, the use of long synchronization preambles could be adopted; however, long preambles significantly increase data overhead. The use of a static, long preamble is particularly unfavorable in applications needing a fast reaction. However, if the synchronization frame is too short, environmental noise could completely waste carrier detection and phase locking. In practice, a right trade-off is needed to set a correct preamble length.

\section{B. Preliminary tests}

In order to better evaluate the impact of these effects on system performance in a real operative scenario, some experimental tests have been performed using the prototype described in the previous section. In each test we considered a different preamble length while maintaining the same transmission parameters. In particular, an office corridor has been chosen as testing area, in two different operative light conditions, namely:

- a Low noise scenario, in which artificial lights are turned off and all windows kept ajar in order to allow a controlled sunlight illumination. In this case, measured illuminance due to sunlight is equal to 25 lux.

- a High noise scenario, in which all artificial lights are turned on and all the windows open, in order to guarantee an high sunlight level in the area. In this case, total measured illuminance in the environment is equal to 1107 lux.

In order to directly verify the effect of environmental changes on the carrier estimation $f_{c}$, its variance $\sigma_{f}^{2}$ has been evaluated during $M=150$ frame transmissions, as indicated in Figure 
3. These tests (1) have been repeated in both light conditions and employing several synchronization lengths $\left(N_{p}\right)$.

$$
\sigma_{f}^{2}=\frac{\sum_{k=1}^{M}\left(f_{c}-\overline{f_{c}}\right)^{2}}{M}
$$

It is evident how a higher level of environmental noise signifi-

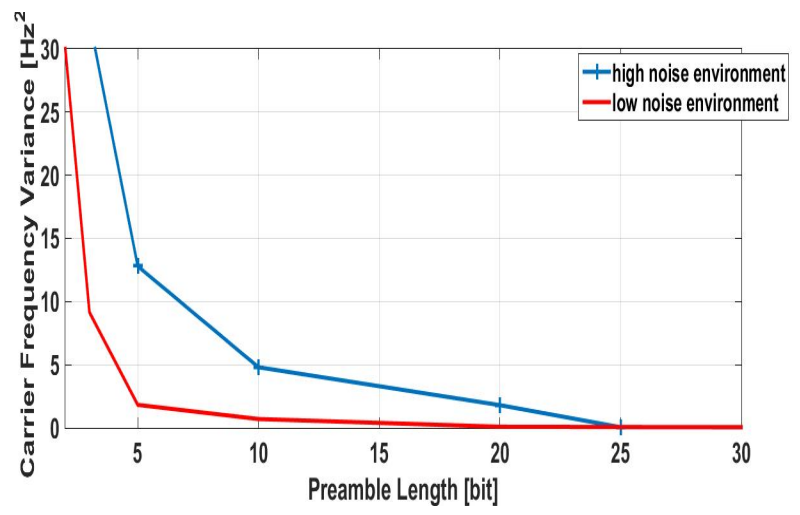

Fig. 3. Variance of recovered central carrier for different values of preamble length.

cantly increases the variance of recovered carrier, unless a significant size of synchronization preamble is $\operatorname{chosen}\left(N_{p} \sim 30\right)$. However, in the case of low noise level, the variance converges quickly to a lower value $\left(N_{p} \sim 5\right)$. Actually, in applications needing small payloads, a long preamble could represent a significant overhead. So, another important parameter to be considered in the analysis is throughput. A unique parameter for evaluating both BER and effective throughput, without involving other system parameters (like transmission rate) has been considered for carrying out a simple analysis without loss of generality. In particular, the instantaneous normalized throughput $T_{\text {inst }}(m)$ (throughput, normalized throughput and goodput refers to the same metric), representing the ratio between the correct received bits in the $m^{\text {th }}$ payload in respect to the overall bits in the frame (including the preamble) has been calculated starting by the instantaneous $B E R_{\text {inst }}(m)$ as in 2 .

$$
T_{\text {inst }}(m)=\left(1-B E R_{\text {inst }}(m)\right) \frac{N}{N_{p}+N}
$$

being $N_{p}$ the length of the current chosen preamble, $N$ the frame payload, fixed a priori and $B E R_{\text {inst }}(m)$ the instantaneous BER at the $m-t h$ payload. In order to analyze the evolution of the throughput after $\mathrm{M}$ transmissions, an average normalized throughput $T(M)$ has been defined in the same way done for average BER, namely

$$
T(M)=\frac{\sum_{m=0}^{M} T_{\text {inst }}(m)}{M+1}
$$

Nevertheless, payload length does not affect recovered carrier variance (and consequently instantaneous BER), in the throughput evaluation it represents an important parameter.
Therefore, a campaign of measurements has been carried out using a fixed payload of $N=2500 \mathrm{bits}$, in both high noise and low noise conditions and shown in Fig. 4 and Fig. 5.

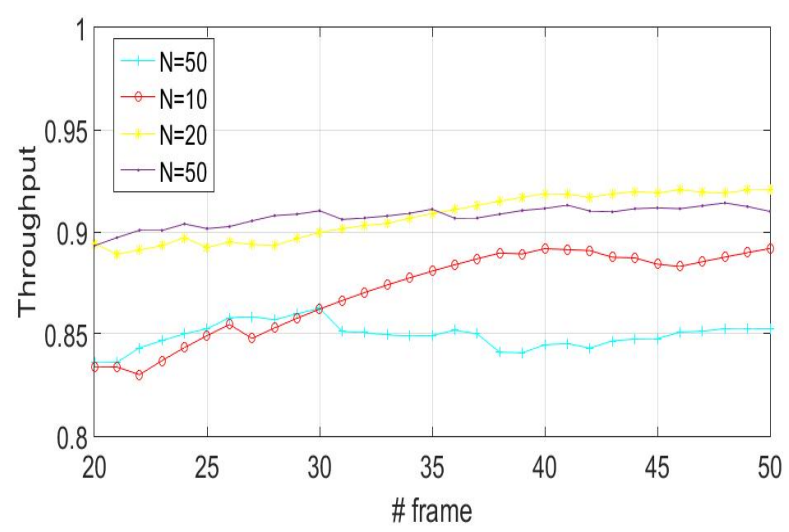

Fig. 4. Measured average normalized throughput for different preamble lengths in high noise environment and 2500 bit payload

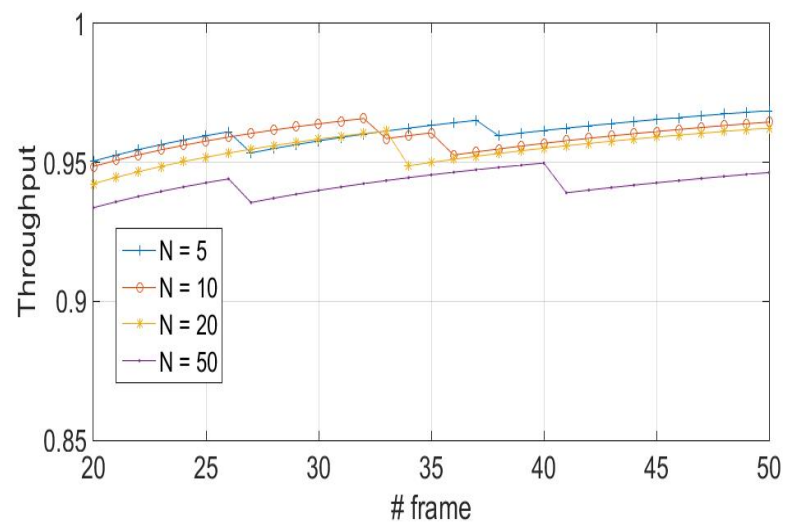

Fig. 5. Measured average normalized throughput for different preamble lengths in low noise environment and 2500 bit payload

\section{Remarks}

Looking at experimental results, the following considerations may be deducted:

- Short synchronization sequences allow good performance in terms of normalized throughput in low noise environmental conditions. In the case of high environmental noise, however, the choice of a short preamble does not allow adequate performances.

- Large synchronization sequences are not efficient in terms of normalized throughput in low environmental conditions, but allow affordable performances in high disturbing light scenes.

- For each environmental scenario, a static selection of the preamble length does not allow the best evolution of the normalizing throughput. Indeed, a real time dynamic selection of synchronization preamble size, based on a 
context aware decision algorithm, may easily improve VLC system performances.

Based on these considerations, a simple intelligent agent has been developed in order to dynamically select, after each received frame, the next preamble size in order to maximize the normalized throughput, on the basis of the evolution of environmental conditions and performance measurements history.

\section{Algorithm}

In order to solve the problem of the dynamical choice of a correct synchronizing preamble, an approach based on the multi-armed bandit problem has been conceived in this work. In our case, as well as several recent applications in different systems [12], Thompson Sampling [13] represents the heuristic for choosing actions that addresses the exploration-exploitation dilemma. We introduce the agent A representing the algorithm defining the actions performed according to previous observations. In particular, we assume $n_{j}$ as the number of times $j^{\text {th }}$ arm has played after $\mathrm{n}$ steps and $\mu_{j}$ to be expected reward of $j^{\text {th }}$ arm.In our case, the played arm is represented by the index of the size of preamble, chosen on a set of 4 possible values, namely $\mathrm{j}=1$ represents $\mathrm{N}=5$ bit, $\mathrm{j}=2$ represents $\mathrm{N}=10$ bit, $\mathrm{j}=3$ represents $\mathrm{N}=20$ bit and $\mathrm{j}=4$ represents $\mathrm{N}$ $=50$ bit). In practice, the preamble size $\mathrm{N}$ is found in average $\mu_{j} n_{j}$ times in $n_{j}$ measurements. In order to directly evaluate both fairness and overhead of the received synchronizing sequence, the criterion trigger we apply in this case is based on the instantaneous normalized throughput evaluation $\mathrm{T}(\mathrm{j})$, as defined in Section III. We assume to have an observation vector collecting $S_{j}$ observations after that we have selected the same size index $j n_{j}$ times. Each size selection is assumed as a Bernoulli distribution with parametric $\mu_{j}$ characterizing the parametric likelihood function for $S_{j}$ as:

$$
p_{j}\left(S_{j} \mid \mu_{j}\right)=\mu_{j}^{t_{j}}\left(1-\mu_{j}\right)^{n_{j}-t_{j}}
$$

where $t_{j}$ is the number of times the best choice in terms of preamble size $j$ has been done. We assume (without loss of generality) that the parameter $\mu_{j}$ is characterized with a Beta distribution as the prior for the distribution. This choice is motivated by the fact that Beta distribution is conjugate prior for the likelihood function in Equation (4). Based on Bayes rule we obtain:

$$
p_{j}\left(\mu_{j} \mid S_{j}\right)=\frac{p_{j}\left(S_{j} \mid \mu_{j}\right) \frac{\Gamma(\alpha+\beta)}{\Gamma(\alpha) \Gamma(\beta)} \mu_{j}^{\alpha-1}\left(1-\mu_{j}\right)^{\beta-1}}{p_{j}\left(S_{j}\right)},
$$

where,

$$
\Gamma(\alpha)=\int_{0}^{\infty} x^{\alpha-1} e^{-x} d x
$$

and $\alpha$ and $\beta$ are the shape parameters of the Beta distribution; we assume (as it is in real world), that we do not have prior information on $\mu_{j}$ and then initial values for $\alpha=\beta=1$ which yields uniform distribution. Substituting (4) in (5) yields,

$$
\begin{array}{r}
p_{j}\left(\mu_{j} \mid S_{j}\right)=\frac{\frac{\Gamma(\alpha+\beta)}{\Gamma(\alpha) \Gamma(\beta)}}{p_{j}\left(S_{j}\right)} \mu_{j}{ }^{t_{j}+\alpha-1}\left(1-\mu_{j}\right)^{n_{j}-t_{j}+\beta-1} . \\
\alpha^{\prime}=t_{j}+\alpha \text { and } \beta^{\prime}=n_{j}-t_{j}+\beta \text { can re-write (7) as: } \\
p_{j}\left(\mu_{j} \mid S_{j}\right)=C \mu_{j}{ }^{\alpha^{\prime}-1}\left(1-\mu_{j}\right)^{\beta^{\prime}-1}
\end{array}
$$

Substituting the normalizing factor $C$ we obtain,

$$
p_{j}\left(\mu_{j} \mid S_{j}\right)=\frac{\Gamma\left(\alpha^{\prime}+\beta^{\prime}\right)}{\Gamma\left(\alpha^{\prime}\right) \Gamma\left(\beta^{\prime}\right)} \mu_{j}{ }^{\alpha^{\prime}-1}\left(1-\mu_{j}\right)^{\beta^{\prime}-1},
$$

which is the beta distribution with parameters $\alpha^{\prime}$ and $\beta^{\prime}$,

$$
p_{j}\left(\mu_{j} \mid S_{j}\right)=\operatorname{beta}\left(\alpha^{\prime}, \beta^{\prime}\right)
$$

Once multi-arm bandit problem was formulated, the following Thompson based algorithm was implemented in our receiving system. The above algorithm has been developed, programming an ad hoc LabView Virtual Instrument for a simple integration in the proposed VLC system.

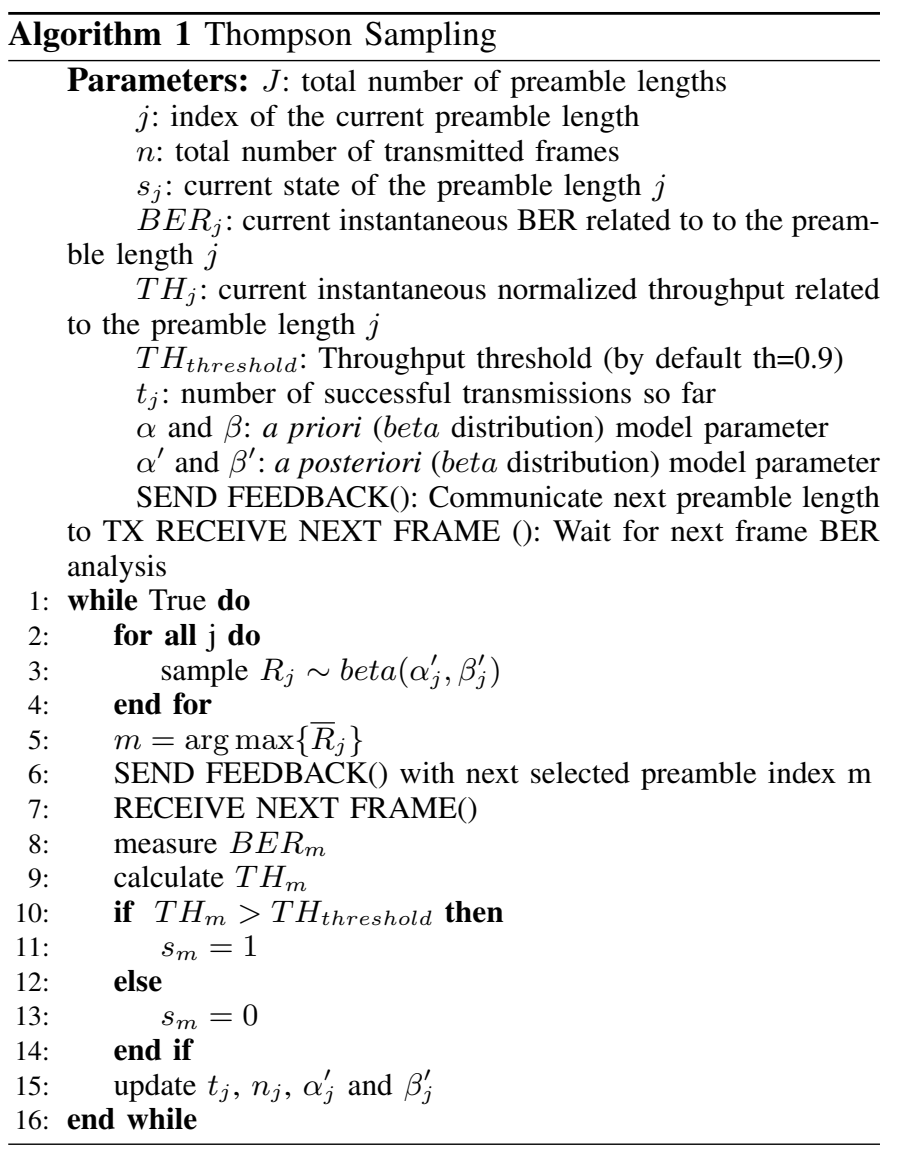

\section{EXPERIMENTAL RESULTS}

\section{A. Preamble Selection}

In order to characterize the behavior of TS algorithm, we have initially carried out experimental tests in the identical conditions of measurement campaign considered in Section III, 
but adding the Intelligent Preamble Selection in our system. For each combination of payload length and environmental light conditions, the first $M=150$ decisions have been recorded and shown in Fig. 6 and 7. Considering a high environmental

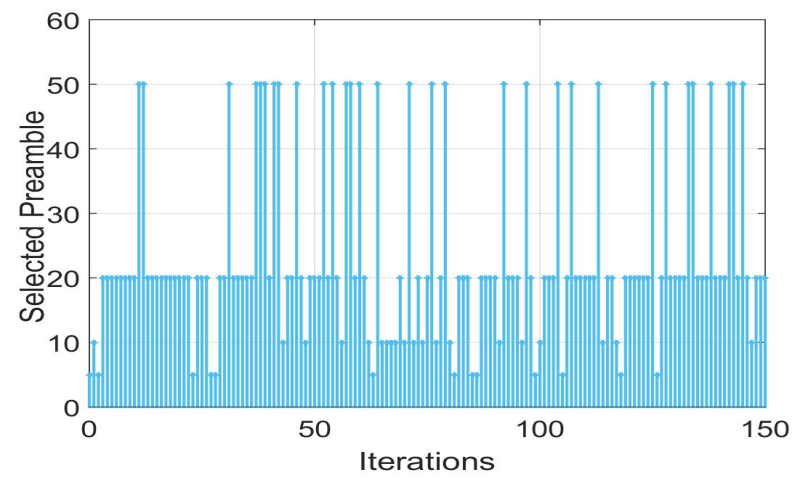

Fig. 6. Preamble length selected by proposed Thompson based algorithm in conditions shown in Fig. 4

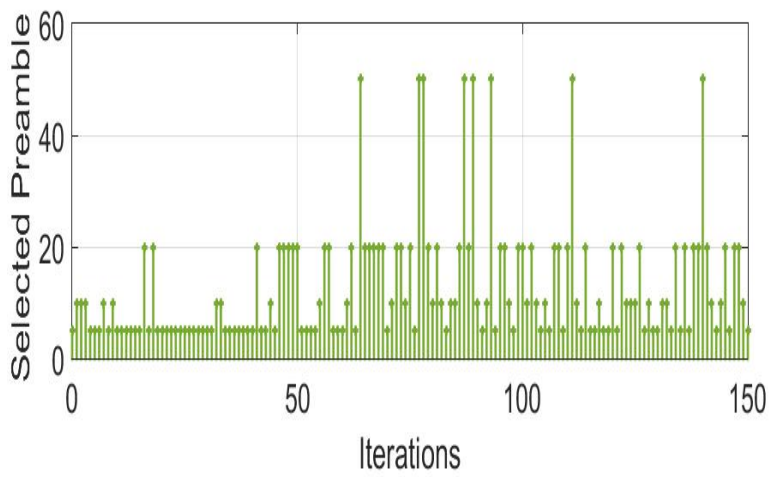

Fig. 7. Preamble length selected by proposed Thompson based algorithm in conditions shown in Fig. 5

noise, low throughput is performed if the smallest preamble is used, while the best performances are for $N_{p}=20$. Small differences can be noticed with the other preamble sizes (Fig. 4). In this case, the best preamble is chosen by the agent 86/150 times (137/150 useful selection if considering also $N_{p}=10$ bit and $N_{p}=50 b i t$ ), while the smallest one just 13/150 times. (6). Maintaining the same payload size but reducing the environmental noise (Fig. 5), the smallest preamble shows the same performances of the intermediate values, but the biggest one shows the worse throughput. In this case, the agent includes the longest preamble, corresponding to the worst choice, only in $8 / 150$ selections (6). In all the cases described above, the most performing preamble size was chosen much more frequently than the other by the intelligent agent. So, even if a reduced set of eligible preamble sizes have been tested, the proposed algorithm correctly follows the evolution of environmental conditions.

\section{B. Throughput Measurements}

For obtaining a further validation of the proposed scheme, a mean between average normalized throughput obtained in the two different environmental light conditions has been performed for each of the static preambles, and compared with the intelligent selection. Results have been shown in Fig. 8 . The best normalized throughput has been achieved

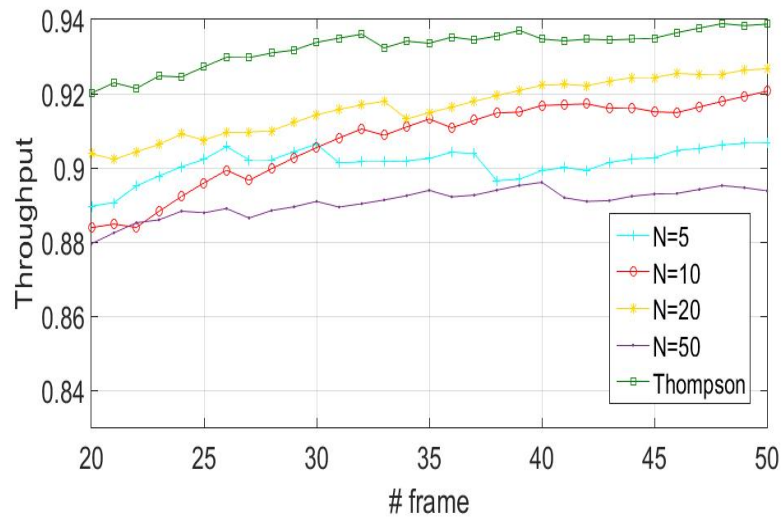

Fig. 8. Measured average normalized throughput comparing different fixed preamble lengths and Thompson sampling selection algorithm for 2500 bit payload, considering a mean of performance in high noise and low noise environments

in the case a Thompson based selection is employed instead of a classical fixed preamble. Normalized throughput obtained by the intelligent agent selection overpasses in each $m^{\text {th }}$ iteration the counterpart obtained by static preamble size, performance of the algorithm are effective for both transitory and converged values. In order to further evaluate the effectiveness of the proposed approach, experimental tests have also been carried out in different conditions of preamble size, payload length (Maximum Transferred Unit - MTU) and environmental light conditions. We have opted to implement the Artificial Intelligence (AI) agent at the receiver side, since it can easily compute the metrics (i.e. BER and goodput) needed to perform the Thompson algorithm. Based on the new selection of preamble length, the receiver send the information to the transmitter on a dedicated control channel. Indeed, the transmitter side is also equipped with a photo-diode for control messages. In the considered scenario, the transmitter and the receiver are approximately $1 \mathrm{~m}$ far away. BER and goodput metrics are both interesting since they provide us a significant characterization of system performance. It is worth to recall that the goodput computed at physical layer is meant as the maximum amount of data that can be transferred in the unit time. Normally, this is an upper bound for the others layers. In Figure 9, we show the results concerning goodput derived from the experimental results averaged on 100000 frames sent. Goodput values shown in Figure 9 are obtained by considering a minimum value of preamble length equal to 20 (Min Preamble), a medium value equal to 500 (Medium Preamble) and the maximum preamble equal to 2000 bits (Maximum Preamble). In the case of minimum 
preamble length a quasi-constant goodput around 0.89 is achieved. The fact that is almost a constant is not surprising, since the minimum payload considered is of 3000bits and 20bits of control impact very little in terms of overhead. Concerning the others two values of preamble, we can notice as their "weight" impacts for different payloads and that an high preamble length can be set only when the payload is "adequate". Another important observation is concerning the values of goodput obtained. Even though 0.89 may appear as an high value, the fact that it is attained at physical layer needs to be considered. With Thompson approach the performance of the system do not vary significantly by varying the payload, by showing the capability of the AI agent to adapt the system to different conditions. Moreover, we are able to obtain a goodput that is at minimum equal to 0.93 . Two key observations can be done. The first one is that even though we manually set the preamble values by ranging on different values, we do not achieve the maximum achievable goodput. The other observation is that the preamble length may impact a lot on the performance of a communication system as we can observe by the minimum goodput achieved. These considerations suggest that the setting of the correct preamble length has to be "automatized" in this type of context.

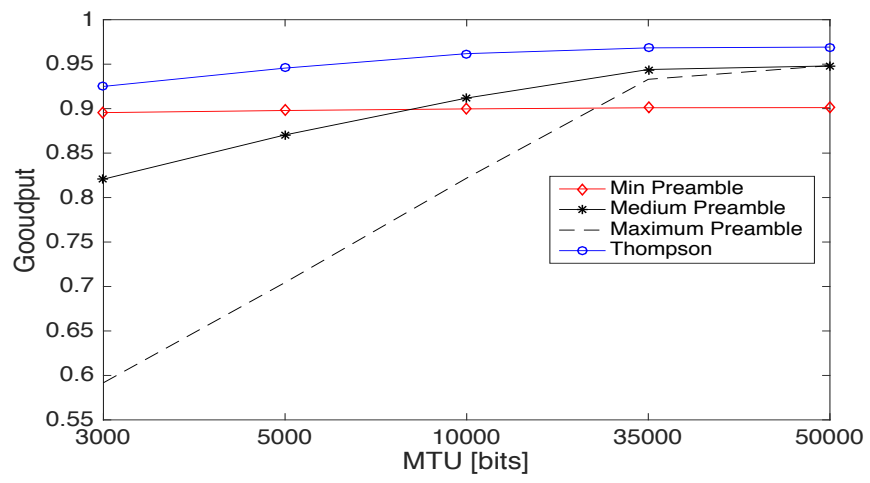

Fig. 9. Goodput evaluation for different values of preamble length.

\section{CONCLUSIONS AND FUTURE PERSPECTIVES}

An adaptive learning approach for calculating variable synchronization frame sizes has been proposed in this paper and applied to VLC systems. An architecture based on low cost hardware, implementing a software defined paradigm, has been implemented in order to demonstrate the need of an adaptive mechanism for real time preamble size setting. Experimental tests demonstrated that preamble size significantly affects the variance of recovered carrier, and, consequently, system performances in terms of Bit Error Ratio and throughput. Preamble size selection has been modeled as a multi-arm bandit problem and Thompson Sampling algorithm has been properly adapted in order to address the exploration-exploitation dilemma. Experimental results show how the agent selects the most appropriate preamble in almost all iterations. If environmental light conditions change, dynamic preamble size setting allow a significant enhancement of performances, compared to a static preamble. In a future extension of this work, different disturbing patterns will be considered in order to further validate the effectiveness of our algorithm, and an extension of this technique to OOK, PSK, PPM and OFDM will be soon provided. The use of a Frequency Shift Keying (FSK) has been considered in this work mainly because of its easier implementation in a low cost architecture, but a future work will deal with system performing different modulation techniques. Our approach could be helpful in real indoor scenarios similar at the one considered in the proposed test (an office corridor), where natural sunlight source penetrates through the windows and changes its intensity during the day. In the scenario we considered, in addition, several artificial lighting devices are turned on and off during normal daily activities. SNR variation during front-end mobility represents an aspect which has not yet been properly faced in the current literature. Errors due to the transitions between shadowed and highly illuminated areas could be properly faced through an intelligent context aware system, especially in possible future outdoor applications.

\section{REFERENCES}

[1] Y. H. Kim, W. A. Cahyadi, Y. H. Chung, "Experimental Demonstration of VLC-Based Vehicle-to-Vehicle Communications Under Fog Conditions," in IEEE Photonics Journal, vol.7, no. 6, Dec. 2015.

[2] C. Wang, H.Y. Yu, Y. J. Zhu, "A Long Distance Underwater Visible Light Communication System With Single Photon Avalanche Diode," in IEEE Photonics Journal, vol. 8, no. 5, Oct. 2016.

[3] Zhuang, Y., Hua, L., Qi, L., Yang, J., Cao, P., Cao, Y., Wu, Y., Thompson, J., Haas, H. "A Survey of Positioning Systems Using Visible LED Lights". in IEEE Commun. Surv. Tutor. 2018.

[4] A. Costanzo, V. Loscri, "Demo: A Context Aware Algorithm for an Adaptive Visible Light Communication System," EWSN, Madrid, Spain, February 2018.

[5] Q. Wang, D. Giustiniano, O. Gnawali, "Low-Cost, Flexible and Open Platform for Visible Light Communication Networks," HotWireless '15. ACM, New York, NY, USA, pp. 31-35, 2015.

[6] S. Schmid, J. Ziegler, G. Corbellini, T. R. Gross, S. Mangold, "Using consumer LED light bulbs for low-cost visible light communication systems," Proceedings of the 1st ACM MobiCom workshop on VLCS '14. ACM, New York, NY, USA,pp. 9-14, 2014.

[7] S. Costanzo, G. Di Massa, A. Costanzo, A. Borgia, A. Raffo, G. Viggiani, P. Versace, "Software-Defined Radar System for Landslides Monitoring, "New Advances in Information Systems and Technologies. Advances in Intelligent Systems and Computing, vol 445. Springer, 2016.

[8] S. Costanzo et al.,"Low-cost radars integrated into a landslide early warning system," New Advances in Information Systems and Technologies. Advances in Intelligent Systems and Computing, vol 354, pp.11-19, Springer, 2016.

[9] A. Cailean, B. Cagneau, L. Chassagne, M. Dimian, V, Popa, “ Novel Receiver Sensor for Visible Light Communications in Automotive Applications,' IEEE Sensors Journal, vol. 15 (8), pp.4632-4639, 2015.

[10] A. Costanzo, V. Loscri, S. Costanzo," Adaptive Dual Color Visible Light Communication (VLC) System", Trends and Advances in Information Systems and Technologies, vol. 2, pp.1478-1487, , Springer, 2016.

[11] A. R. Ndjiongue, H. C. Ferreira, T. M. N. Ngatched, " Visible Light Communications (VLC) Technology," Wiley Encyclopedia of Electrical and Electronics Engineering,pp. 1-15, 2015.

[12] V. Toldov, L. Clavier, V. Loscri, N. Mitton,“ A Thompson sampling approach to channel exploration-exploitation problem in multihop cognitive radio networks,'IEEE 27th PIMRC, Valencia, Sept. 2016.

[13] W. R. Thompson, "On the likelihood that one unknown probability exceeds another in view of the evidence of two samples," Biometrika, vol. 25(34), pp 285-294, 1933. 